Preprint DSF-T-1/95; DAMTP/95-15

gr-qc/9503040

\title{
Inflationary Cosmology from Noncommutative Geometry
}

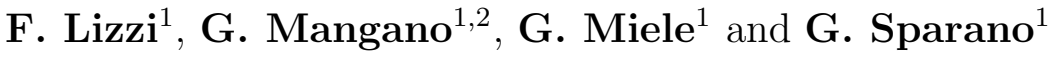 \\ ${ }^{1}$ Dipartimento di Scienze Fisiche, Università di Napoli - Federico II -, and INFN \\ Sezione di Napoli, Mostra D’Oltremare Pad. 20, 80125, Napoli, Italy \\ 2 Department of Applied Mathematics and Theoretical Physics, \\ Silver Street, CB3 9EW, Cambridge, United Kingdom
}

\begin{abstract}
In the framework of the Connes-Lott model based on noncommutative geometry, the basic features of a gauge theory in the presence of gravity are reviewed, in order to show the possible physical relevance of this scheme for inflationary cosmology. These models naturally contain at least two scalar fields, interacting with each other whenever more than one fermion generation is assumed. In this paper we propose to investigate the behaviour of these two fields (one of which represents the distance between the copies of a two-sheeted space-time) in the early stages of the universe evolution. In particular the simplest abelian model, which preserves the main characteristics of more complicate gauge theories, is considered and the corresponding inflationary dynamics is studied. We find that a chaotic inflation is naturally favoured, leading to a field configuration in which no symmetry breaking occurs and the final distance between the two sheets of space-time is smaller the greater the number of $e$-fold in each sheet.
\end{abstract}

To be published in the International Journal of Modern Physics A 


\section{Introduction}

The early universe is certainly the best laboratory to probe the structure of space and time at very short distances, and during this period of the universe, inflation plays a central role in solving important problems occurring in standard cosmology. It is usually assumed that the dynamics of scalar fields is responsible for inflation, by producing an effective cosmological constant term in the Einstein equations. This leads, as well-known, to a quasi de Sitter epoch during which the universe scale factor grows almost exponentially. The knowledge of quantum field effects, in this era, is particularly relevant because they are the origin of the subsequent structure of the present universe. Thus, all models in which some scalar fields are present can be used in principle to describe inflation, provided that the corresponding dynamics is appropriate.

In this respect, the recently proposed gauge models inspired by noncommutative geometry are a relevant example of theories in which scalar fields and corresponding interaction potentials emerge naturally from geometry. In this paper we study, for a wide class of these models, the predictions on the resulting inflationary scenarios.

The programme of noncommutative geometry [1] shifts the stress from space and time, traditionally seen as a geometrical ensemble of points, to the (commutative) algebra of continuous complex valued functions defined on it, with the perspective of possible noncommutative generalizations. One of the most interesting and promising products of this programme is the formulation of the electroweak standard model by Connes and Lott [2, [3], generalized to include Grand Unified Theories in [4]. Loosely speaking, this formulation is based on a doubling of space-time, considered as a two sheeted manifold. The algebra of continuous functions and the Hilbert space of fields is thus doubled as well. The nontrivial feature of the theory is that the Dirac operator, as well as the gauge potential (connection), have some nondiagonal elements, which couple the two sheets of space-time. These are classical scalar fields: one is related to the component of the metric in the discrete direction, and thus to the distance between the two sheets of space-time, and the others are Higgs fields, responsible for the breaking of the symmetry. We will discuss in more detail the field content of the theory in the first sections. 
The action is as usual the square of the curvature of the connection. As the Dirac operator contains the metric structure of a manifold, the presence of this coupling gives a theory of gravity as well [5, 6]. By simply postulating that at the level of two-sheeted algebra the action is the square of the curvature one obtains the Einstein-Hilbert YangMills action containing naturally an Higgs potential.

Interestingly, at the classical level this scheme generally predicts relations between the physical masses of particles, such as the ratio of the top quark mass over the Higgs mass, and fixes in some ranges the values of relevant constants appearing in the electroweak standard model [7].

The aims of such a programme are, in the long run, the prediction of physical masses or other experimentally testable quantities, and even more the creation of a unique framework to include gravity. In this initial stage, however, the stress is more on the understanding of how the noncommutative structure of space-time might account for the already known features of this (exceptionally well working) electroweak model how we understand it presently. This understanding, combined with the tools of noncommutative geometry, should then provide the framework for the unveiling of deeper symmetries of the model, and the possibilities to go further towards the unification with gravity.

At the moment all studies have been performed at the classical level, and quantization can only be achieved with the usual field theory tools. This quantization, however, although it does not respect the relations between the coupling constants [8], under the renormalization flow does not change considerably these relations over various orders of magnitude of the scale. A fully satisfactory achievement of the programme would be a quantization made on the level of the algebra, and therefore intrinsically related to noncommutative geometry. This remains one of the main challenges of the programme.

On more phenomenological grounds, there is also a problem of scales which requires further studies. The scalar field which is part of the Riemann connection and is related to the distance between the two sheets of space-time, pertaining to the intrinsic structure of the latter, has as a natural scale, the Planck length. On the other side, according to the Connes and Lott model [2], at low energy this distance must be the inverse of the top quark mass, which is several order of magnitude larger than the Planck length, to correctly 
reproduce the electroweak standard model. We would like to stress that an important feature of our work is to look at the distance as undergoing a dynamical evolution. It remains a challenging programme to study the dynamics responsible for the subsequent evolution of the distance down to the electroweak scale.

In this paper we study the scalar dynamics at the Planck energy since we are interested in the cosmological implications of the noncommutative scenarios. As we already noticed the natural epoch in which the scalar fields, related to the connections in the discrete direction, may play a fundamental role is the inflationary one. Therefore, we have studied, for the simplest abelian theory, all the relevant characteristic which a good inflationary model should satisfy: a quite large $e$-fold number $N$, large enough $\left(N \simeq 10^{2}\right)$ to solve the flatness problem of standard cosmology; an efficient reheating stage, which consists in the transfer of the enormous energy density stored in the inflaton fields into entropy density for the relativistic degrees of freedom.

It is worth-while stressing that, although our analysis has been performed for the simplest abelian model, the results are general enough to be looked upon as indicating a typical behaviour of a noncommutative inspired gauge model. This is because it already contains all the key features of more realistic dynamics based on some non-abelian gauge symmetry group.

We cannot say in fact what the full gauge theory of fields and particles was at epochs shortly after Planck time, and therefore we cannot decide the specific model of noncommutative geometry to be used, namely which Hilbert space and Dirac operator we must use. Nevertheless all considered models present at least the fields mentioned above, namely two scalar fields which are the discrete components of the gauge and gravitational connections.

This paper is organized as follows: in section 2 we review the model of Yang-Mills theory plus gravity in noncommutative geometry, and obtain for the abelian case the expression for the bosonic lagrangian. Section 3 is devoted to the study of the scalar potential and to the related inflationary dynamics. The one-loop effective potential is computed in the adiabatic approximation and the results obtained solving the semiclassical scalar field equations of motion are discussed. Finally we analyze, at one-loop level, the 
reheating mechanism by introducing the coupling of the scalars to the fermionic degrees of freedom. It is shown that this mechanism is efficient enough to make the transition from the vacuum energy density to the radiation dominated universe. In section 4 we give our conclusions and outlooks.

\section{General relativity and gauge theories in noncom- mutative geometry}

In this section the construction of a Einstein-Yang-Mills theory in noncommutative geometry is briefly reviewed. The results, are then applied to the abelian case. The reason of this choice is that, as far as the scalar fields are concerned, the nonabelian aspect is practically irrelevant (apart of the different irreducible representations to which the scalar fields belong to) on the resulting inflationary dynamics. Thus, in this scenario, the abelian theory not only represents a toy model for which it is interesting to study the cosmological implications, but it also contains the essential ingredients of any more complicate, but physically more relevant, gauge theory inspired by noncommutative theory.

\subsection{Gravity}

In order to obtain the Einstein-Hilbert action [5] we first introduce the notion of a $K$-cycle. A $K$-cycle 且, 因, 田, is a triple $(\mathcal{A}, \mathcal{H}, D)$ where: $\mathcal{A}$ is a $*$-algebra unitarily represented on the Hilbert space $\mathcal{H}$ in terms of bounded operators, and $D$ is an unbounded selfadjoint operator with compact resolvent such that the commutator with any element of the algebra is bounded. This operator is the generalized Dirac operator. The usual concepts of Riemannian geometry are now derived by the $K$-cycle. The general idea is the following: the Riemannian metric is defined in terms of the $K$-cycle as a hermitian structure $<., .>$ on $\Omega_{D}^{1}(\mathcal{A})$, which is the space of 1 -forms on $\mathcal{A}[1,3]$ and thus is the noncommutative analog of the cotangent bundle. As far as the notion of connection is concerned, it is useful to think of a connection $\nabla$ on a fiber bundle as a linear map

$$
\nabla: E \longrightarrow \Lambda^{1}(\mathcal{M}) \otimes E
$$

satisfying the Leibniz rule, where $E$ is the space of sections of the fiber bundle and $\Lambda^{1}(\mathcal{M})$ is the space of 1 -forms on the base manifold $\mathcal{M}$. In the case of a linear connection on 
$\mathcal{M}$, observing that we are interested in forms rather than vector fields, the fiber bundle is taken to be the cotangent bundle. Furthermore, if on $\mathcal{M}$ there is a metric $g^{E}$ (the index $E$ hereafter denotes the Euclidean version), a Levi-Civita connection is a linear connection satisfying the requirements

$$
\begin{aligned}
\nabla g^{E} & =0 \\
T(\nabla) \equiv d-P \circ \nabla & =0
\end{aligned}
$$

where $T(\nabla)$ is the torsion corresponding to the connection $\nabla$, and $P$ maps the tensor product of two 1-forms into their exterior product. The noncommutative notion of a Levi-Civita connection will then be given by 2.1 with $E$ and $\Lambda^{1}$ both replaced by $\Omega_{D}^{1}(\mathcal{A})$

$$
\nabla: \Omega_{D}^{1}(\mathcal{A}) \longrightarrow \Omega_{D}^{1}(\mathcal{A}) \otimes \Omega_{D}^{1}(\mathcal{A})
$$

Of the two conditions $(2.2)$ and (2.3), the former is naturally implemented by requiring

$$
d<\xi, \eta>=<\nabla \xi, \eta>-<\xi, \nabla \eta>
$$

whereas the latter can be straightforwardly used. The action of $\nabla$ can be extended to the whole universal exterior algebra allowing the definition of the curvature $R(\nabla)$ as

$$
R(\nabla)=-\nabla^{2}
$$

We will consider gravity on $\overline{\mathcal{M}}=\mathcal{M} \times Z_{2}$ where $\mathcal{M}$ is the Euclidean space-time. On $\overline{\mathcal{M}}$ the algebra $\mathcal{A}$ is taken as the product of two copies of $C^{\infty}$ functions on $\mathcal{M}$

$$
\mathcal{A}=C^{\infty}(\mathcal{M}) \oplus C^{\infty}(\mathcal{M})
$$

$\mathcal{A}$ acts diagonally on the Hilbert space $\mathcal{H}$, whose Dirac operator is

$$
D=\left(\begin{array}{cc}
\nabla & \gamma_{5} \phi \\
\gamma_{5} \phi & \nabla
\end{array}\right)
$$

Let us introduce in $\Omega_{D}^{1}(\mathcal{A})$ an orthonormal basis $e^{N}$ such that

$$
\begin{aligned}
& <e^{N}, e^{M}>=\delta^{N M}, \\
& e^{a}=\left(\begin{array}{cc}
\gamma^{a} & 0 \\
0 & \gamma^{a}
\end{array}\right),
\end{aligned}
$$




$$
e^{5}=\left(\begin{array}{cc}
0 & \gamma_{5} \\
-\gamma_{5} & 0
\end{array}\right),
$$

where $N, M=1, \ldots, 5 ; \gamma^{a}=\gamma^{\mu} e_{\mu}^{a}$ and $e_{\mu}^{a} e_{\nu}^{a}=g_{\mu \nu}^{E}$, with $a, \mu=1, \ldots, 4$ 冈. The covariant derivative operator $\nabla$ is characterized by the matrix of 1 -forms $\omega_{M}^{N}$ defined by

$$
\nabla e^{N}=-\omega_{M}^{N} \otimes e^{M}
$$

Analogously the curvature is characterized by the 2 -forms $R_{M}^{N}$ given by

$$
R(\nabla) e^{N}=R_{M}^{N} \otimes e^{M}=\left(d \omega_{M}^{N}+\omega_{P}^{N} \omega_{M}^{P}\right) \otimes e^{M}
$$

The forms $\omega_{M}^{N}$ can be written as

$$
\omega_{M}^{N}=\left(\begin{array}{cc}
\gamma^{\mu} \omega_{1 \mu M}^{N} & \gamma_{5} \phi l_{M}^{N} \\
\gamma_{5} \phi\left(l^{T}\right)_{M}^{N} & \gamma^{\mu} \omega_{2 \mu M}^{N}
\end{array}\right)
$$

By imposing the conditions (2.2) and (2.3) one can see that $\omega_{M}^{N}$ can be written in terms of the Levi-Civita connection $\omega_{\mu b}^{a}$ on $\mathcal{M}$, of the metric field $\phi$, and of the auxiliary fields $l_{M}^{N}$ [5]. The Euclidean Einstein-Hilbert action is

$$
I_{E-H}^{E}=(32 \pi G)^{-1} \int_{\mathcal{M}} \operatorname{Tr}\left[R_{M}^{N} e^{M}\left(e_{N}\right)^{*}\right]-2 \Lambda \int_{\mathcal{M}} \sqrt{-g} d^{4} x
$$

Eliminating the auxiliary fields and continuing to the Lorentzian signature one obtains

$$
I_{E-H}=\int_{\mathcal{M}} \sqrt{-g}\left(-\frac{1}{16 \pi G} \mathcal{R}-2 \Lambda+\frac{1}{2} \partial_{\mu} \sigma \partial^{\mu} \sigma\right) d^{4} x
$$

where $\mathcal{R}$ stands for the scalar curvature of $\mathcal{M}$, and $\sigma$ is a real scalar field such that $\phi=\mu \exp (-k \sigma)\left(\mu\right.$ is an arbitrary mass and $\left.k \equiv \sqrt{4 \pi G}=\sqrt{4 \pi / M_{P l}^{2}}\right)$.

\subsection{Gauge theory}

In this subsection we use the method just developed to add to the gravity action the Yang-Mills action for a nonabelian gauge group [2, 4]. For simplicity let us consider the case $S U_{L}(n) \otimes S U_{R}(m) \otimes U_{B}(1)$ with $L$ and $R$ standing for lefthanded and righthanded chirality, respectively. In this case the space will have the same structure as in last section, $\overline{\mathcal{M}} \equiv \mathcal{M} \times Z_{2}=\mathcal{M}_{L} \cup \mathcal{M}_{R}$, with $\mathcal{M}$ being a smooth spin manifold.

\footnotetext{
${ }^{*}$ Remind that the Euclidean Dirac matrices $\gamma_{\mu}$ and $\gamma_{5}$ obey to the following algebra: $\gamma_{\mu}^{\dagger}=-\gamma_{\mu}$, $\left\{\gamma_{\mu}, \gamma_{\nu}\right\}=-2 g_{\mu \nu}^{E}, \gamma_{5}^{\dagger}=\gamma_{5}$
} 
We will consider the $K$-cycle for which $\mathcal{H}$ is the Hilbert space made of fermions $\psi_{L}^{b}$ and $\psi_{R}^{b}$ belonging to $n$-plet and $m$-plet of the gauge groups $U_{L}(n)$ and $U_{R}(m)$, respectively. Hence a vector $\psi$ of $\mathcal{H}$ will have the form

$$
\Psi=\left(\begin{array}{c}
\psi_{L}^{b} \\
\psi_{R}^{b}
\end{array}\right)
$$

$b=1, . ., n_{g}$ standing for the family number, with $n_{g}$ the number of generations. Thus the Hilbert space results to be $\mathcal{H}=L^{2}\left(S_{L}\right) \oplus L^{2}\left(S_{R}\right)$ with $S_{L(R)}$ denoting a bundle on $\mathcal{M}_{L(R)}$ of multiplets spinors under the gauge group $U_{L}(n)\left(U_{R}(m)\right)$. The $*$-algebra, $\mathcal{A}$, of bounded operators acting on $\mathcal{H}$, is taken as

$$
\mathcal{A}=C^{\infty}(\mathcal{M}) \otimes\left(U_{L}(n) \oplus U_{R}(m)\right)
$$

An arbitrary element of $\mathcal{A}, a$, results to be a $(n+m) \times(n+m)$ matrix

$$
a=\left(\begin{array}{cc}
a^{L} & 0 \\
0 & a^{R}
\end{array}\right)
$$

where $a_{L}$ and $a_{R}$ are two unitary $n \times n$ and $m \times m$ matrices of functions, respectively. The Euclidean Dirac operator, $D$, is given by

$$
D \equiv\left(\begin{array}{cc}
\not \partial \otimes \mathbb{I}_{n} \otimes \mathbb{I}_{n_{g}} & \gamma_{5} \phi \otimes M \otimes K \\
\gamma_{5} \phi \otimes M^{\dagger} \otimes K^{\dagger} & \not \partial \otimes \mathbb{I}_{m} \otimes \mathbb{I}_{n_{g}}
\end{array}\right),
$$

with $M$ an $n \times m$ mass matrix, and $K$ a matrix responsible for a family number mixing.

In this formalism the gauge potentials $A_{\mu}^{L(R)}$, defined on the manifold $\mathcal{M}_{L(R)}$, are contained in the 1-form

$$
\rho \equiv \sum_{j} a_{j}\left[D, b_{j}\right]=\left(\begin{array}{cc}
A^{L} & \gamma_{5} \Phi \otimes K \\
\gamma_{5} \Phi^{\dagger} \otimes K^{\dagger} & A^{R}
\end{array}\right)
$$

where in (2.21) we have used the following definitions

$$
\begin{aligned}
A^{L(R)} & =\gamma^{\mu} A_{\mu}^{L(R)} \equiv \gamma^{\mu} \sum_{j} a_{j}^{L(R)} \partial_{\mu} b_{j}^{L(R)} \\
\Phi & =\phi \sum_{j} a_{j}^{L}\left(M b_{j}^{R}-b_{j}^{L} M\right)
\end{aligned}
$$

By imposing the condition

$$
\sum_{j} a_{j}^{L(R)} b_{j}^{L(R)}=1
$$


it follows that $A_{\mu}^{L(R)}$ defined in (2.22) are antihermitian $n \times n(m \times m)$ matrices, which are connected to the generators of $U_{L}(n)\left(U_{R}(m)\right)$. Thus in order to obtain the gauge group $S U_{L}(n) \otimes S U_{R}(m) \otimes U_{B}(1)$ one has to require a graded trace condition on $\rho$, namely $\operatorname{Tr}\left(A_{\mu}^{L}\right)=\operatorname{Tr}\left(A_{\mu}^{R}\right)$. We can express $A_{\mu}^{L(R)}$ in terms of the generators ${ }^{\dagger}$ of $S U_{L}(n)\left(S U_{R}(m)\right)$ $\mathcal{T}_{p}^{L}\left(\mathcal{T}_{q}^{R}\right)\left(p=1, . ., n^{2}-1 ; q=1, . ., m^{2}-1\right)$

$$
\begin{aligned}
& A_{\mu}^{L} \equiv-\frac{i}{\sqrt{2}} g_{L} \mathcal{T}_{p}^{L} W_{\mu}^{p L}-\frac{i}{\sqrt{2}} g_{B} \sqrt{\frac{m}{n(n+m)}} \mathbb{I}_{n} B_{\mu} \\
& A_{\mu}^{R} \equiv-\frac{i}{\sqrt{2}} g_{R} \mathcal{T}_{q}^{R} W_{\mu}^{q R}-\frac{i}{\sqrt{2}} g_{B} \sqrt{\frac{n}{m(n+m)}} \mathbb{I}_{m} B_{\mu}
\end{aligned}
$$

where $W_{\mu}^{p L}\left(W_{\mu}^{q R}\right)$ are the corresponding hermitian gauge potentials and $B_{\mu}$ is the $U_{B}(1)$ gauge field. The Euclidean Yang-Mills action, as well-known, is given by the following expression

$$
I_{Y-M}^{E}=\frac{1}{8 \mathcal{N}} \int d^{4} x \sqrt{g^{E}} \operatorname{Tr}\left(\theta^{2}\right)
$$

where the 2 -form $\theta \equiv d \rho+\rho^{2}, d \rho=\sum_{j}\left[D, a_{j}\right]\left[D, b_{j}\right]$, and $\mathcal{N}$ is a normalization constant to be determined. From the above considerations we get the following expressions for the matrix elements of $\theta$

$$
\begin{aligned}
\theta_{L L} & =\frac{1}{2} \gamma^{\mu \nu} G_{\mu \nu}^{L}+\nabla_{L}^{\mu} A_{\mu}^{L}-\sum_{j} a_{j}^{L} \square b_{j}^{L}+\left(\Phi \Phi^{\dagger}-\phi^{2} \sum_{j} a_{j}^{L} M M^{\dagger} b_{j}^{L}\right) K K^{\dagger}, \\
\theta_{R R} & =\frac{1}{2} \gamma^{\mu \nu} G_{\mu \nu}^{R}+\nabla_{R}^{\mu} A_{\mu}^{R}-\sum_{j} a_{j}^{R} \square b_{j}^{R}+\left(\Phi^{\dagger} \Phi-\phi^{2} \sum_{j} a_{j}^{R} M^{\dagger} M b_{j}^{R}\right) K^{\dagger} K, \\
\theta_{L R} & =\gamma_{5}\left[-\not \partial \Phi-k \Phi \not \partial \sigma+\Phi A^{R}-\not A^{L} \Phi\right] K, \\
\theta_{R L} & =\gamma_{5}\left[-\not \partial \Phi^{\dagger}-k \Phi^{\dagger} \not \partial \sigma-A^{R} \Phi^{\dagger}+\Phi^{\dagger} A^{L}\right] K^{\dagger} .
\end{aligned}
$$

Note that, for sake of simplicity, in Eqs. (2.28) we have shifted the field $\Phi$ with respect to the definition (2.23), and we have denoted with $\gamma^{\mu \nu} \equiv\left[\gamma^{\mu}, \gamma^{\nu}\right] / 2$. Moreover, given the covariant derivative (2.12), we define the gauge covariant derivative as $\nabla_{L(R)}^{\mu} \equiv \nabla^{\mu}+A_{L(R)}^{\mu}$ and the Yang-Mills tensor $G_{\mu \nu}^{L(R)} \equiv \nabla_{\mu}^{L(R)} A_{\nu}^{L(R)}-\nabla_{\nu}^{L(R)} A_{\mu}^{L(R)}$. It is now convenient to introduce the following auxiliary fields

$$
X_{L(R)} \equiv-\nabla_{L(R)}^{\mu} A_{\mu}^{L(R)}+\sum_{j} a_{j}^{L(R)} \square b_{j}^{L(R)}
$$

\footnotetext{
${ }^{\dagger}$ For the generators we assume the normalization conditions $\operatorname{Tr}\left(\mathcal{T}_{p}^{L} \mathcal{T}_{p^{\prime}}^{L}\right)=2 \delta_{p p^{\prime}}$ and $\operatorname{Tr}\left(\mathcal{T}_{q}^{R} \mathcal{T}_{q^{\prime}}^{R}\right)=$ $2 \delta_{q q^{\prime}}$
} 


$$
\begin{aligned}
Y_{L} & \equiv \sum_{j} a_{j}^{L} M M^{\dagger} b_{j}^{L}, \\
Y_{R} & \equiv \sum_{j} a_{j}^{R} M^{\dagger} M b_{j}^{R} .
\end{aligned}
$$

Thus, substituting (2.29)-(2.31) in (2.28) we get

$$
\begin{aligned}
\theta_{L L} & =\frac{1}{2} \gamma^{\mu \nu} G_{\mu \nu}^{L}-X_{L}+\left(\Phi \Phi^{\dagger}-\phi^{2} Y_{L}\right) K K^{\dagger}, \\
\theta_{R R} & =\frac{1}{2} \gamma^{\mu \nu} G_{\mu \nu}^{R}-X_{R}+\left(\Phi^{\dagger} \Phi-\phi^{2} Y_{R}\right) K^{\dagger} K, \\
\theta_{L R} & =\gamma_{5}\left[-\not \partial \Phi-k \Phi \not \partial \sigma+\Phi A^{R}-A^{L} \Phi\right] K, \\
\theta_{R L} & \left.=\gamma_{5}\left[-\not \partial \Phi^{\dagger}-k \Phi^{\dagger} \not \partial \sigma-A^{R} \Phi^{\dagger}+\Phi^{\dagger} \not A^{L}\right)\right] K^{\dagger} .
\end{aligned}
$$

The Euclidean bosonic lagrangian density corresponding to the Yang-Mills contribution (2.27) is then given by

$$
\begin{aligned}
\mathcal{L}_{B}^{E} & =\frac{\sqrt{g^{E}}}{\mathcal{N}}\left\{\frac{1}{2} \operatorname{Tr}\left[K K^{\dagger}\left(\Phi \Phi^{\dagger}-\phi^{2} Y_{L}\right)-X_{L}\right]^{2}+\frac{1}{2} \operatorname{Tr}\left[K^{\dagger} K\left(\Phi^{\dagger} \Phi-\phi^{2} Y_{R}\right)-X_{R}\right]^{2}\right. \\
& -\operatorname{Tr}\left(K K^{\dagger}\right) \operatorname{Tr}\left[\left(\partial_{\mu} \Phi-\Phi A_{\mu}^{R}+A_{\mu}^{L} \Phi+k \Phi \partial_{\mu} \sigma\right)\right. \\
& \left.\left.\times\left(\partial^{\mu} \Phi^{\dagger}+A^{R \mu} \Phi^{\dagger}-\Phi^{\dagger} A^{L \mu}+k \Phi^{\dagger} \partial^{\mu} \sigma\right)\right]-\frac{1}{4} n_{g} \operatorname{Tr}\left[G_{L}^{\mu \nu} G_{\mu \nu}^{L}+G_{R}^{\mu \nu} G_{\mu \nu}^{R}\right]\right\} \cdot
\end{aligned}
$$

The continuation to the Lorentzian signature is easily obtained by substituting $\mathcal{L}_{B}^{E} \Rightarrow-\mathcal{L}_{B}$ and $x_{4} \Rightarrow i t$. Replacing in (2.33) the fields $A_{\mu}^{L(R)}$ with the physical gauge potentials $W_{\mu}^{p L}$, $W_{\mu}^{q R}$ and $B_{\mu}$ we get

$$
\begin{aligned}
\mathcal{L}_{B} & =\frac{\sqrt{-g}}{\mathcal{N}}\left\{-\frac{1}{2} \operatorname{Tr}\left[K K^{\dagger}\left(\Phi \Phi^{\dagger}-\phi^{2} Y_{L}\right)-X_{L}\right]^{2}-\frac{1}{2} \operatorname{Tr}\left[K^{\dagger} K\left(\Phi^{\dagger} \Phi-\phi^{2} Y_{R}\right)-X_{R}\right]^{2}\right. \\
& +\operatorname{Tr}\left(K^{\dagger} K\right) \operatorname{Tr}\left[\left(\mathcal{D}^{\mu} \Phi\right)^{\dagger}\left(\mathcal{D}_{\mu} \Phi\right)+k \partial_{\mu}\left(\Phi^{\dagger} \Phi\right) \partial^{\mu} \sigma+k^{2} \Phi^{\dagger} \Phi \partial_{\mu} \sigma \partial^{\mu} \sigma\right] \\
& \left.-\frac{1}{4} n_{g}\left(g_{L}^{2} W_{p L}^{\mu \nu} W_{\mu \nu}^{p L}+g_{R}^{2} W_{q R}^{\mu \nu} W_{\mu \nu}^{q R}+g_{B}^{2} B_{\mu \nu} B^{\mu \nu}\right)\right\}
\end{aligned}
$$

where $W_{\mu \nu}^{q L(R)}$ and $B_{\mu \nu}$ are the Yang-Mills tensors for left (right) and $U_{B}(1)$ gauge fields, respectively, and

$$
\mathcal{D}_{\mu} \Phi \equiv \partial_{\mu} \Phi+i \frac{g_{R}}{\sqrt{2}} \Phi \mathcal{T}_{q}^{R} W_{\mu}^{q R}-i \frac{g_{L}}{\sqrt{2}} \mathcal{T}_{p}^{L} \Phi W_{\mu}^{p L}+i \frac{g_{B}}{\sqrt{2}} \frac{n-m}{\sqrt{n m(n+m)}} B_{\mu} \Phi
$$

Note that, in order to have in Eq.(2.34) the usual kinetic terms for the gauge degrees of freedom it is necessary to put $g_{L}=g_{R}=g_{B}$. To obtain the final expression for the bosonic action we need to eliminate from (2.34) the contribution coming from the 
auxiliary fields $Y_{L(R)}$ and $X_{L(R)}$, and this is performed by varying the lagrangian with respect to them and substituting the resulting constraints in (2.34). It is immediately clear from this procedure that the presence, in the final expression of $\mathcal{L}_{B}$, of potential terms for the $\Phi$ field is strongly depending on the form of the $M$ matrix. To be more precise, it is connected to the possibility, for the particular choice made for $M$, of not having independent auxiliary fields.

\subsection{A simple abelian model}

As clear from the previous sections, the scalar fields content of a noncommutative Einstein -Yang-Mills theory is essentially independent of the particular gauge group chosen. Any models will have at least a real $\sigma$ field coming from gravity and one or more $\Phi$ Higgs fields. This simple consideration suggests to consider the simplest gauge theory, that is $n=m=1$, in order to study the resulting cosmological implications. The point we wish to make is that the corresponding results will reliably sketch the inflationary scenario of any more complicate but more realistic GUT model.

According to Eq. (2.20), the new Dirac operator in the Euclidean space results to be

$$
D \equiv\left(\begin{array}{cc}
\not \partial \otimes \mathbb{I}_{n_{g}} & \gamma_{5} \phi \otimes K \\
\gamma_{5} \phi \otimes K^{\dagger} & \not \partial \otimes \mathbb{I}_{n_{g}}
\end{array}\right)
$$

Consequently, following the previous analysis we get the expression for the matrix elements of $\theta$, which read

$$
\begin{aligned}
\theta_{L L} & =-\frac{i}{4} g_{B} \gamma^{\mu \nu} B_{\mu \nu}-X_{L}+\left(|\Phi|^{2}-\mu^{2} \exp (-2 k \sigma)\right) K K^{\dagger} \\
\theta_{R R} & =-\frac{i}{4} g_{B} \gamma^{\mu \nu} B_{\mu \nu}-X_{R}+\left(|\Phi|^{2}-\mu^{2} \exp (-2 k \sigma)\right) K^{\dagger} K \\
\theta_{L R} & =-\gamma_{5}(\not \partial \Phi+k \Phi \not \partial \sigma) \\
\theta_{R L} & =-\gamma_{5}\left(\not \partial \Phi^{*}+k \Phi^{*} \not \partial \sigma\right)
\end{aligned}
$$

where $\Phi$ is an usual complex field. Thus we get the bosonic lagrangian in Lorentzian space-time which is

$$
\begin{aligned}
\mathcal{L}_{B} & =\frac{\sqrt{-g}}{\mathcal{N}}\left\{-\frac{1}{4} n_{g} g_{B}^{2} B_{\mu \nu} B^{\mu \nu}-\left[\operatorname{Tr}\left(K^{\dagger} K\right)^{2}-\frac{\left(\operatorname{Tr}\left(K^{\dagger} K\right)\right)^{2}}{n_{g}}\right]\left[|\Phi|^{2}-\mu^{2} \exp (-2 k \sigma)\right]^{2}\right. \\
& \left.+\operatorname{Tr}\left(K^{\dagger} K\right) \quad\left[\partial^{\mu} \Phi^{*} \partial_{\mu} \Phi+k \partial_{\mu}|\Phi|^{2} \partial^{\mu} \sigma+k^{2}|\Phi|^{2} \partial_{\mu} \sigma \partial^{\mu} \sigma\right]\right\} .
\end{aligned}
$$


From (2.38) it is clear that only for $K^{\dagger} K \neq \beta \mathbb{I}_{n_{g}}$ the potential term for the Higgs field $\Phi$ is preserved. With $\mathcal{N}=n_{g} g_{B}{ }^{2}$ we obtain the usual lagrangian. Furthermore, redefining $\Phi \equiv \varphi g_{B}\left[n_{g} / \operatorname{Tr}\left(K^{\dagger} K\right)\right]^{1 / 2}$, and finally $\mu \equiv m g_{B}\left[n_{g} / \operatorname{Tr}\left(K^{\dagger} K\right)\right]^{1 / 2}$ we get

$$
\begin{aligned}
\mathcal{L}_{B} & =\sqrt{-g}\left\{-\frac{1}{4} B_{\mu \nu} B^{\mu \nu}-\frac{\lambda}{4 !}\left[|\varphi|^{2}-m^{2} \exp (-2 k \sigma)\right]^{2}-V_{0}\right. \\
& \left.+\left[\partial^{\mu} \varphi \partial_{\mu} \varphi^{*}+k \partial_{\mu}|\varphi|^{2} \partial^{\mu} \sigma+\left(\frac{1}{2}+k^{2}|\varphi|^{2}\right) \partial_{\mu} \sigma \partial^{\mu} \sigma\right]\right\} .
\end{aligned}
$$

In (2.39) the kinetic term of the $\sigma$-field and the constant cosmological term appearing in (2.16), $V_{0}$, are also included, and the definition

$$
\lambda \equiv 6 g_{B}^{2}\left[\frac{n_{g} \operatorname{Tr}\left(K^{\dagger} K\right)^{2}}{\left(\operatorname{Tr}\left(K^{\dagger} K\right)\right)^{2}}-1\right] \geq 0
$$

is used. From Eq. (2.39) one easily gets the tree-level potential for the $\varphi$ and $\sigma$ fields $V(\sigma, \varphi)$

$$
V(\sigma, \varphi)=V_{0}+\frac{\lambda}{4 !}\left[|\varphi|^{2}-m^{2} \exp (-2 k \sigma)\right]^{2}
$$

This potential has its minimum for $|\varphi|^{2}=m^{2} \exp (-2 k \sigma)$, which is a condition which does not fix both $|\varphi|$ and $\sigma$ uniquely. The situation changes if one assumes $\sigma$ to be still a classical field, and consider the one-loop corrections to $V(\sigma, \varphi)$ coming from the quantum fluctuations of $\varphi$ [9, 10]. This assumption is consistent with the physical scenario we want to describe, since the inflationary epoch is assumed to be described by Einstein gravity (classical theory). In our case this means that the classical Einstein theory can be applied to the doubled manifold, so $\sigma$, which is the metric component in the discrete dimension, has to be a pure classical field with no quantum fluctuations. On the contrary, $\varphi$ is an usual scalar field and thus for it we will consider quantum fluctuations.

\section{Inflation from scalar dynamics}

To study the inflation induced in the early universe by the dynamics of $\sigma$ and $\varphi$, one needs to solve the equations of motion for these fields starting from reasonable initial conditions. In this section we will discuss the evolution of this coupled system showing how this evolution corresponds, for a natural choice of initial conditions, to an inflationary phase. 


\subsection{The effective potential}

The Higgs field $\varphi$, contrary to $\sigma$ which we will treat as a pure classical field, can be expanded around its background configuration as

$$
\varphi=\frac{\varphi_{c}}{\sqrt{2}}+\frac{\delta \varphi_{1}+i \delta \varphi_{2}}{\sqrt{2}}
$$

where the background configuration $\varphi_{c} / \sqrt{2} \equiv\langle\varphi\rangle$ can always be chosen real using the $U(1)$ gauge symmetry of the theory. With $\delta \varphi_{i}$ we denote the independent quantum fluctuations, which satisfy $\left\langle\delta \varphi_{i}\right\rangle=0$. By substituting (3.42) in (2.39), we get the following equations of motion for $\varphi_{c}, \sigma$, and $\delta \varphi_{i}$

$$
\begin{gathered}
\square \varphi_{c}+k \varphi_{c} \square \sigma-k^{2} \varphi_{c} \partial_{\mu} \sigma \partial^{\mu} \sigma+\frac{\lambda}{24}\left[\varphi_{c}^{2}-2 m^{2} \exp (-2 k \sigma)+\left\langle 3 \delta \varphi_{1}^{2}+\delta \varphi_{2}^{2}\right\rangle\right] \varphi_{c}=0 \\
{\left[1+k^{2}\left(\varphi_{c}^{2}+\left\langle\delta \varphi_{1}^{2}+\delta \varphi_{2}^{2}\right\rangle\right)\right] \square \sigma+2 k^{2} \varphi_{c} \partial_{\mu} \varphi_{c} \partial^{\mu} \sigma+k \partial_{\mu} \varphi_{c} \partial^{\mu} \varphi_{c}+k \varphi_{c} \square \varphi_{c}} \\
+\frac{\lambda}{12} k m^{2} \exp (-2 k \sigma)\left[\varphi_{c}^{2}-2 m^{2} \exp (-2 k \sigma)+\left\langle\delta \varphi_{1}^{2}+\delta \varphi_{2}^{2}\right\rangle\right]=0 \\
\square \delta \varphi_{1}+\frac{\lambda}{24}\left[3 \varphi_{c}^{2}-2 m^{2} \exp (-2 k \sigma)\right] \delta \varphi_{1}+\frac{\lambda}{24}\left[\delta \varphi_{1}^{3}+\delta \varphi_{1} \delta \varphi_{2}^{2}\right] \\
+\frac{\lambda}{24} \varphi_{c}\left[3\left(\delta \varphi_{1}^{2}-\left\langle\delta \varphi_{1}^{2}\right\rangle\right)+\left(\delta \varphi_{2}^{2}-\left\langle\delta \varphi_{2}^{2}\right\rangle\right)\right]=0 \\
\square \delta \varphi_{2}+\frac{\lambda}{24}\left[\varphi_{c}^{2}-2 m^{2} \exp (-2 k \sigma)\right] \delta \varphi_{2}+\frac{\lambda}{24}\left[\delta \varphi_{2}^{3}+\delta \varphi_{1}^{2} \delta \varphi_{2}+2 \varphi_{c} \delta \varphi_{1} \delta \varphi_{2}\right]=0
\end{gathered}
$$

The quantities $\left\langle\delta \varphi_{i}^{2}\right\rangle$ in Eqs. (3.43) and (3.44) account for the one-loop corrections to the effective potential. Note that in the quantum fluctuation equations of motion (3.45) and (3.46) the equation of motion for $\varphi_{c}$ has been used, and according to the adiabatic approximation [10], we have neglected all terms proportional to derivatives of the background fields.

In order to linearize the last two equations with respect to $\delta \varphi_{i}$, one can estimate the self-interaction terms of these fields in the mean-field approximation. According to this ansatz, the quadratic and cubic terms of $\delta \varphi_{i}$ result to be $\delta \varphi_{i}^{3}=3\left\langle\delta \varphi_{i}^{2}\right\rangle \delta \varphi_{i}, \delta \varphi_{i}^{2}=\left\langle\delta \varphi_{i}^{2}\right\rangle$ 
and $\delta \varphi_{1} \delta \varphi_{2}=\left\langle\delta \varphi_{1} \delta \varphi_{2}\right\rangle=0$ respectively. In particular the vanishing of the $\delta \varphi_{1} \delta \varphi_{2}$ correlator can be easily understood from the explicit expression of the interaction term of the fluctuations; from (2.41)

$$
V_{\delta \varphi_{1} \delta \varphi_{2}}=\frac{\lambda}{96}\left[4 \varphi_{c}\left(\delta \varphi_{1}^{3}+\delta \varphi_{1} \delta \varphi_{2}^{2}\right)+2 \delta \varphi_{1}^{2} \delta \varphi_{2}^{2}+\delta \varphi_{1}^{4}+\delta \varphi_{2}^{4}\right]
$$

one get the conclusion that, at all orders in perturbation theory, there are no contributions to graphs with an odd number of $\delta \varphi_{2}$ external lines so, in particular, $\left\langle\delta \varphi_{1} \delta \varphi_{2}\right\rangle=0$. We also notice that all expectation values of cubic terms, like $\left\langle\delta \varphi_{1}^{3}\right\rangle$, do not contribute to (3.45) and (3.46). By taking the expectation value of these equations, in fact, and using the condition $\left\langle\delta \varphi_{i}\right\rangle=0$, it follows that the sum of all these contributions identically vanishes.

The linearized expressions for Eqs. (3.45) and (3.46) take then the form

$$
\begin{aligned}
& \square \delta \varphi_{1}+\frac{\lambda}{24}\left[3 \varphi_{c}^{2}-2 m^{2} \exp (-2 k \sigma)+\left\langle 3 \delta \varphi_{1}^{2}+\delta \varphi_{2}^{2}\right\rangle\right] \delta \varphi_{1}=0 \\
& \square \delta \varphi_{2}+\frac{\lambda}{24}\left[\varphi_{c}^{2}-2 m^{2} \exp (-2 k \sigma)+\left\langle\delta \varphi_{1}^{2}+3 \delta \varphi_{2}^{2}\right\rangle\right] \delta \varphi_{2}=0
\end{aligned}
$$

By following [10], at zero-th order in the adiabatic expansion, we get the gap equations for $\left\langle\delta \varphi_{i}^{2}\right\rangle$

$$
\begin{aligned}
\left\langle\delta \varphi_{1}^{2}\right\rangle & =\frac{1}{8 \pi^{2}}\left\{Q^{2}+\left[\frac{\lambda}{48}\left(3 \varphi_{c}^{2}-2 m^{2} \exp (-2 k \sigma)+3\left\langle\delta \varphi_{1}^{2}\right\rangle+\left\langle\delta \varphi_{2}^{2}\right\rangle\right)\right]\right. \\
& \left.\times\left[\log \left|\frac{\lambda\left[3 \varphi_{c}^{2}-2 m^{2} \exp (-2 k \sigma)+3\left\langle\delta \varphi_{1}^{2}\right\rangle+\left\langle\delta \varphi_{2}^{2}\right\rangle\right]}{96 Q^{2}}\right|+1\right]\right\} \\
\left\langle\delta \varphi_{2}^{2}\right\rangle & =\frac{1}{8 \pi^{2}}\left\{Q^{2}+\left[\frac{\lambda}{48}\left(\varphi_{c}^{2}-2 m^{2} \exp (-2 k \sigma)+\left\langle\delta \varphi_{1}^{2}\right\rangle+3\left\langle\delta \varphi_{2}^{2}\right\rangle\right)\right]\right. \\
& \left.\times\left[\log \left|\frac{\lambda\left[\varphi_{c}^{2}-2 m^{2} \exp (-2 k \sigma)+\left\langle\delta \varphi_{1}^{2}\right\rangle+3\left\langle\delta \varphi_{2}^{2}\right\rangle\right]}{96 Q^{2}}\right|+1\right]\right\}
\end{aligned}
$$

Note that for simplicity, we have neglected the contribution of the coupling of $\varphi$ with the scalar curvature $R$, because it rapidly becomes negligible. Thus the Eqs. (3.50) and (3.51) are just the flat space corrections à la Coleman-Weinberg [9].

The parameter $Q$ is the ultraviolet cut-off which, representing the energy upper bound for the validity of our model, will be assumed in the following of the order of the Planck 
mass. These two expressions can be solved by iteration. In the following we will only consider the zero-th order approximation, which already gives results correct at order $\lambda$

$$
\begin{gathered}
\left\langle\delta \varphi_{1}^{2}\right\rangle=\frac{1}{8 \pi^{2}}\left\{Q^{2}+\left[\frac{\lambda}{48}\left(3 \varphi_{c}^{2}-2 m^{2} \exp (-2 k \sigma)+\frac{Q^{2}}{2 \pi^{2}}\right)\right]\right. \\
\times\left[\log \left|\frac{\lambda\left[3 \varphi_{c}^{2}-2 m^{2} \exp (-2 k \sigma)+Q^{2} /\left(2 \pi^{2}\right)\right]}{96 Q^{2}}\right|+1\right], \\
\left\langle\delta \varphi_{2}^{2}\right\rangle=\frac{1}{8 \pi^{2}}\left\{Q^{2}+\left[\frac{\lambda}{48}\left(\varphi_{c}^{2}-2 m^{2} \exp (-2 k \sigma)+\frac{Q^{2}}{2 \pi^{2}}\right)\right]\right. \\
\left.\times\left[\log \left|\frac{\lambda\left[\varphi_{c}^{2}-2 m^{2} \exp (-2 k \sigma)+Q^{2} /\left(2 \pi^{2}\right)\right]}{96 Q^{2}}\right|+1\right]\right\} .
\end{gathered}
$$

From Eqs. (3.48) and (3.49) one reads the square of the effective masses for the quantum fluctuations

$$
\begin{aligned}
& \Omega_{1}^{2}=\frac{\lambda}{24}\left[3 \varphi_{c}^{2}-2 m^{2} \exp (-2 k \sigma)+\frac{Q^{2}}{2 \pi^{2}}\right], \\
& \Omega_{2}^{2}=\frac{\lambda}{24}\left[\varphi_{c}^{2}-2 m^{2} \exp (-2 k \sigma)+\frac{Q^{2}}{2 \pi^{2}}\right]
\end{aligned}
$$

As it always occurs in scalar field theories, these masses get contributions from the highest scale involved in the model, which in this case is the Planck mass.

By virtue of Eqs. (3.52) and (3.53) one can determine the quantum fluctuation contributions to (3.43) and (3.44)

$$
\begin{gathered}
\square \varphi_{c}+k \varphi_{c} \square \sigma-k^{2} \varphi_{c} \partial_{\mu} \sigma \partial^{\mu} \sigma+\frac{\delta V_{e f f}}{\delta \varphi_{c}}=0 \\
{\left[1+k^{2}\left(\varphi_{c}^{2}+\frac{Q^{2}}{4 \pi^{2}}\right)\right] \square \sigma+2 k^{2} \varphi_{c} \partial_{\mu} \varphi_{c} \partial^{\mu} \sigma+k \partial_{\mu} \varphi_{c} \partial^{\mu} \varphi_{c}+k \varphi_{c} \square \varphi_{c}+\frac{\delta V_{e f f}}{\delta \sigma}=0}
\end{gathered}
$$

where the effective potential $V_{\text {eff }}\left(\sigma, \varphi_{c}\right)$, in terms of the definitions (3.54) and (3.55), results to be

$$
\begin{aligned}
V_{e f f}\left(\sigma, \varphi_{c}\right) & =V_{0}+\frac{\lambda}{96}\left[\varphi_{c}^{2}-2 m^{2} \exp (-2 k \sigma)\right]^{2}+\frac{\lambda Q^{2}}{96 \pi^{2}}\left[\varphi_{c}^{2}-m^{2} \exp (-2 k \sigma)\right] \\
& +\frac{1}{64 \pi^{2}}\left\{\Omega_{1}^{4}\left[\log \left|\frac{\Omega_{1}^{2}}{4 Q^{2}}\right|+\frac{1}{2}\right]+\Omega_{2}^{4}\left[\log \left|\frac{\Omega_{2}^{2}}{4 Q^{2}}\right|+\frac{1}{2}\right]\right\} .
\end{aligned}
$$

Notice that in the factor in front of $\square \sigma$ in Eq. (3.57) we have only considered the zero-th order term in the $\lambda$ expansion; this is to be consistent with the fact that, according to 
the adiabatic expansion, we have neglected in Eqs. (3.45) and (3.46) the terms order $\lambda$, which are proportional to the derivative of the $\sigma$ field.

The quadratic and logarithmic divergences in $V_{\text {eff }}$ when $Q^{2} \rightarrow \infty$ are usually removed by the renormalization procedure, namely by a redefinition of the mass and the coupling constant. This is always possible for a potential of the form (2.41) if the term $m^{2} \exp (-2 k \sigma)$ is just a constant. In our case, however, the tree level expectation value of the $\varphi$ field depends on the dynamical field $\sigma$. If one assumes that the latter evolves quite rapidly with respect to $\varphi$, once $\sigma$ has reached a stationary point $\sigma_{m}$, the dynamics of the $\varphi$ becomes the one governed by the usual mexican hat potential

$$
V(\sigma, \varphi)=V_{0}+\frac{\lambda}{4 !}\left[|\varphi|^{2}-m^{2} \exp \left(-2 k \sigma_{m}\right)\right]^{2}
$$

which can be renormalized in order to take into account the effects of quantum fluctuations. In the general case we are dealing with, where the simultaneous evolution of $\varphi$ and $\sigma$ is considered, our model is not renormalizable, i.e. both divergences cannot simply absorbed in the parameters of the lagrangian density. This fact is not surprising, since the $\sigma$ field is related to gravity in the discrete direction, and still we are far from a renormalizable theory where gravitational fields are considered as dynamical degrees of freedom. In the following we will look at (3.58) as an effective potential and we will fix the cut-off, as already mentioned, at the order of the Planck mass scale, which is the natural one at which the field dynamics takes place. We will see, by numerically solving the one-loop equations of motions, that in fact the evolution of $\sigma$ is quite fast due to the exponential nature of its potential. However, the largest contribution to the $e$-fold number just comes from the time interval during which $\sigma$ reaches its stationary point, so the $\sigma$ dynamics, as far as the inflationary dynamics is concerned, is particularly relevant.

The study of the minima of the effective potential (3.58) can be analytically performed in a simple way if we consider only terms up to the first order in $\lambda$ : in this case the only minimum is located at the point $\varphi_{c, m}=0, \sigma_{m}=(1 / 2 k) \log \left(8 \pi^{2} m^{2} / Q^{2}\right)$. By requiring that the effective cosmological constant vanishes at the absolute minimum we therefore get $V_{0}=\lambda Q^{4} /\left(1536 \pi^{4}\right)$. We have numerically checked that the shift in the true minimum due to the order $\lambda^{2}$ logarithmic terms is of few percents for $\lambda \sim .1$, which will be the largest 
value we will use in the following in order to have a reliable perturbative treatment of the scalar dynamics.

In Figure 1. the effective potential for a choice of parameters, namely $Q=10 M_{P l}$, $m=1 M_{P l}$ and $\lambda=0.1$, is shown.

\subsection{The equations of motion}

Since we are interested in the cosmological implications of the scalar dynamics, and in particular in the possibility to get a reasonable inflationary stage, we choose a spatially flat Friedmann-Robertson-Walker (FRW) universe, whose metric has the simple form

$$
d s^{2}=d t^{2}-a^{2}(t) d \vec{x}^{2}
$$

and assume homogeneous field configurations, namely $\sigma=\sigma(t), \varphi_{c}=\varphi_{c}(t)$.

Under this assumption, the equations of motion Eqs.(3.56)-(3.57), once put in normal form, read

$$
\begin{gathered}
\ddot{\sigma}+3 H \dot{\sigma}+\left(1+\frac{k^{2} Q^{2}}{4 \pi^{2}}\right)^{-1}\left[k\left(\dot{\varphi}_{c}+k \varphi_{c} \dot{\sigma}\right)^{2}+\frac{\delta V_{\text {eff }}}{\delta \sigma}-k \varphi_{c} \frac{\delta V_{\text {eff }}}{\delta \varphi_{c}}\right]=0, \\
\ddot{\varphi}_{c}+3 H \dot{\varphi}_{c}-k \varphi_{c}\left(1+\frac{k^{2} Q^{2}}{4 \pi^{2}}\right)^{-1}\left[k\left(\dot{\varphi}_{c}+k \varphi_{c} \dot{\sigma}\right)^{2}+\frac{\delta V_{\text {eff }}}{\delta \sigma}-k \varphi_{c} \frac{\delta V_{\text {eff }}}{\delta \varphi_{c}}\right] \\
-k^{2} \varphi_{c} \dot{\sigma}^{2}+\frac{\delta V_{\text {eff }}}{\delta \varphi_{c}}=0,
\end{gathered}
$$

where $H$ is the Hubble parameter, defined as

$$
H \equiv\left(\frac{\dot{a}}{a}\right)=\sqrt{\frac{2}{3}} k\left\{\frac{\dot{\varphi}_{c}^{2}}{2}+k \varphi_{c} \dot{\varphi}_{c} \dot{\sigma}+\frac{1}{2}\left[1+k^{2}\left(\varphi_{c}^{2}+\frac{Q^{2}}{4 \pi^{2}}\right)\right] \dot{\sigma}^{2}+V_{e f f}\left(\sigma, \varphi_{c}\right)\right\}^{1 / 2}
$$

We will fix the initial conditions for $\sigma$ and $\varphi_{c}$ by requiring that the corresponding initial value for the energy density is of the order of $M_{P l}^{4}$, with vanishing kinetic terms. Moreover, we also require that the initial distance between the two sheets, $d_{5}(0)$, is equal to the Planck length, to be consistent with the idea that our model is able to describe the evolution of the universe just after the quantum epoch for gravity. Since all dynamics only depends on the combination $d_{5}^{-1}=m \exp (-k \sigma)$, and $m$ can be reexpressed in terms of $d_{5}(0)$, we get $d_{5}^{-1}=d_{5}^{-1}(0) \exp [-k(\sigma-\sigma(0))]$. This allows, by shifting $\sigma \rightarrow \sigma+\sigma(0)$, to take $\sigma(0)=0$ as initial condition without loss of generality, since all the other terms 
in the equation of motion are invariant under this transformation. Consequently, the requirement on the initial energy density fixes, up to an inessential sign, $\varphi_{c}(0)$.

For the free parameters of $V_{\text {eff }}, \lambda$ and $Q$, we have considered the following ranges: $\lambda \in[0.01,0.1]$, which ensures the reliability of the perturbative approach, and $Q \gtrsim M_{P l}$. The latter choice is, of course, based on the reasonable assumption that in the initial stage the only typical mass scale is $M_{P l}$.

The set of coupled equations (3.61)-(3.63) has been numerically solved. We will here sketch the main features of the $\sigma-\varphi_{c}$ motion: a more detailed analysis is reported in the next section, where also the reheating epoch is considered.

The dynamics is characterized by two main behaviours: in a first phase the scalar fields evolves towards the minimum $\sigma=\sigma_{m}, \varphi \sim 0$ (see section 3.1). During this rolling down, as in the chaotic scenarios, the scale factor rapidly grows, faster the larger is the value of the cut-off parameter $Q$. It is interesting to note that, since the stationary point for $\varphi$ differs from zero for terms of the order $\lambda^{2}$, the $U(1)$ symmetry is broken quite weakly. In particular, if the choice for $Q$ satisfies the condition $Q<2 \pi M_{P l}$ it is easy to see that the $\varphi_{c}$ field undergoes an inverse phase transition. At $t=0$ in fact, having chosen $d_{5}(0)=M_{P l}^{-1}$, $\Omega_{i}^{2}\left(\varphi_{c}=0\right)<0$, showing that we are in a broken symmetry phase: in these conditions, in fact, the minimum at fixed $\sigma=\sigma(0)=0$ is located at $\varphi_{c, m}^{2}=2 M_{P l}^{2}-Q^{2} / 2 \pi^{2}$. When $d_{5}$, due to the $\sigma$ evolution, reaches the critical value $d_{5}^{c r}=2 \pi / Q>d_{5}(0)$ the symmetry is restored, up to terms order $\lambda^{2}$ due to the logarithmic terms in $V_{\text {eff }}$, since the true minimum becomes $\varphi_{c}=0$. Differently, if $Q \geq 2 \pi M_{P l}$ all the $\varphi_{c}$ evolution take place in the unbroken phase, since in this case $d_{5}$ always remains above the critical value.

Once the rolling down stops, the value of $\sigma$ remains practically fixed at $\sigma_{m}$, while $\varphi_{c}$ starts oscillating around the equilibrium configuration. This phase is extremely important for reheating since, as well known, during these oscillations the scalar fields may transfer their energy density to the incoherent gas of $\delta \varphi_{i}$ quanta and, more generally, to other particles via decay processes. 


\subsection{The Reheating}

It is well known that the necessary last stage of the inflationary dynamics is the so-called reheating era. During this phase the energy density stored in the scalar sector is released to the relativistic degrees of freedom via decay processes. This epoch is at least as important as the truly inflationary one, since it guarantees the end of the exponential-like growth of the scale factor and the reheating of the universe, which will then continue to expand as in a radiation dominated era. It is usually stated that the inflaton field configuration, once it has reached its minimum, can be regarded as a condensate of the corresponding massive quanta, which would decay in other light particles. Such a coupling is assumed to give rise to an effective term of the form $\Gamma \dot{\Phi}$, where $\Gamma$ is the decay rate, which is put by hand into the inflaton equation of motion. It has been shown however that this procedure is unsatisfactory [11, 12]. A better approximation consists in deducing the friction terms from the one-loop effects of quantum fluctuations at first order in the adiabatic expansion, as done in [10] and [12]. This procedure would imply the appearance of terms of the form $\Gamma H \Phi$ in the inflaton equation of motion and, moreover, that the mass of the $\Phi$ quanta should be considered as time dependent. We will restrict ourselves in the following to this approach; this will give a correct qualitative description for small times, but, as any other perturbative computation, it would fail for large times. This is due, in particular, to resonance phenomena which enhance the order $\hbar$ term amplitude over the tree amplitude, so one should make use of a Hartree or mean field technique similar to the one discussed in the previous section [12]. This would lead to a set of gap equations to be solved at higher order in the adiabatic expansion. However it is outside the aim of this paper a detailed analysis of the reheating of the universe: the final stage of the inflationary era, of course, strongly depends on the particular model one assumes, as well on the way the inflaton field is coupled to the relativistic degrees of freedom. What we would like to show is simply that even the simplest abelian model may give rise to an efficient transfer of the energy density from the coupled system of scalar field configurations $\sigma-\varphi_{c}$ to radiation.

To this end we will describe the reheating phase as due to decay processes of the $\varphi$ field into fermionic degrees of freedom $\Psi$ (see eq. (2.17)), due to the interaction term $\operatorname{Tr}[\bar{\Psi}(D+\rho) \Psi]$, where $D$ is defined by (2.36) and $\rho$ is the abelian version of the gauge 
potential matrix (2.21). These processes lead to an energy transfer from the coherent classical configuration, whose evolution drives the inflationary expansion of the universe, to the incoherent gas of $\Psi$ quanta: if the latter starts to dominate the energy density of the universe, the inflation stops, since their equation of state relating the energy density $\rho_{R}$ and the pressure $p_{R}$ satisfies the condition $\rho_{R}+3 p_{R}=4 \rho_{R}>0$.

The interaction term can be written in the usual Yukawa form

$$
\mathcal{L}_{Y u k}=-f a(t)^{4} \varphi \overline{\psi_{L}} \psi_{R}+\text { h.c. } .
$$

Using the background splitting (3.42) we have

$$
\mathcal{L}_{Y u k}=-\frac{f}{\sqrt{2}} a(t)^{4}\left[\varphi_{c} \overline{\psi_{L}} \psi_{R}+\left(\delta \varphi_{1}+i \delta \varphi_{2}\right) \overline{\psi_{L}} \psi_{R}\right]+\text { h.c. }
$$

implying that the fermions acquire an effective mass $m_{\Psi}=f \varphi_{c} / \sqrt{2}$. For the decay of the $\delta \varphi_{i}$ quanta into a fermion-antifermion pair to be possible the scalar particle masses should be greater than twice the fermion mass. The corresponding decay probabilities in the $\delta \varphi_{i}$ rest frame can be simply evaluated in the Born approximation and result to be

$$
\Gamma_{i} \equiv \Gamma\left(\delta \varphi_{i} \rightarrow \Psi \bar{\Psi}\right)=n_{f} \frac{f^{2}}{16 \pi} \Omega_{i} \Theta\left(\Omega_{i}-2 m_{\Psi}\right) \quad i=1,2
$$

where $n_{f} \sim 10^{2}$ is the number of independent decay channels. In the general case of a non-abelian gauge theory, one should also consider interaction terms of the scalar field $\Phi$ with the gauge bosons, see (2.34). This would increase the number of possible decay channels for $\Phi$ and the efficiency of the entropy release to the relativistic degrees of freedom. However the analysis for the simple abelian case will be general enough to show how the model may also quite naturally account for the end of inflation.

The $\varphi_{c}$ and $\sigma$ equations of motion modify due to the presence of the interaction terms with the fermionic field: the expectation value of the fluctuation field squared $\left\langle\delta \varphi_{i}^{2}\right\rangle$ should in fact include the interaction with the $\Psi$ degrees of freedom. Following [10, to which for brevity we refer for the detailed calculation, one finds

$$
\left\langle\delta \varphi_{i}^{2}\right\rangle=\left\langle\delta \varphi_{i}^{2}\right\rangle_{0}+\frac{1}{256 \pi}\left[\frac{\dot{\Omega}_{i}}{\Omega_{i}}+H\right] \Gamma_{i}
$$

where with $\left\langle\delta \varphi_{i}^{2}\right\rangle_{0}$ we have denoted the corresponding result in absence of the interaction term (3.65), which has been quoted in (3.52) and (3.53). Using these expressions we can 
compute the equations of motion for $\sigma$ and $\varphi_{c}$; as before, inserting (3.67) into (3.43) and (3.44) and passing to normal form

$$
\begin{gathered}
\ddot{\sigma}+3 H \dot{\sigma}+\left(1+\frac{k^{2} Q^{2}}{4 \pi^{2}}\right)^{-1}\left[k\left(\dot{\varphi}_{c}+k \varphi_{c} \dot{\sigma}\right)^{2}+\frac{\delta V_{e f f}}{\delta \sigma}-k \varphi_{c} \frac{\delta V_{e f f}}{\delta \varphi_{c}}\right. \\
\left.+\gamma_{\sigma}-k \varphi_{c} \gamma_{\varphi_{c}}\right]=0 \\
\ddot{\varphi}_{c}+3 H \dot{\varphi}_{c}-k \varphi_{c}\left(1+\frac{k^{2} Q^{2}}{4 \pi^{2}}\right)^{-1}\left[k\left(\dot{\varphi}_{c}+k \varphi_{c} \dot{\sigma}\right)^{2}+\frac{\delta V_{e f f}}{\delta \sigma}-k \varphi_{c} \frac{\delta V_{e f f}}{\delta \varphi_{c}}\right. \\
\left.+\gamma_{\sigma}-k \varphi_{c} \gamma_{\varphi_{c}}\right]-k^{2} \varphi_{c} \dot{\sigma}^{2}+\frac{\delta V_{e f f}}{\delta \varphi_{c}}+\gamma_{\varphi_{c}}=0
\end{gathered} .
$$

With $\gamma_{\varphi_{c}}$ and $\gamma_{\sigma}$ we have denoted the following quantities

$$
\begin{aligned}
\gamma_{\varphi_{c}} & =\frac{\lambda \varphi_{c}}{6144 \pi}\left[\left(3 \Gamma_{1}+\Gamma_{2}\right) H+3 \frac{\dot{\Omega}_{1}}{\Omega_{1}} \Gamma_{1}+\frac{\dot{\Omega}_{2}}{\Omega_{2}} \Gamma_{2}\right] \\
\gamma_{\sigma} & =\frac{\lambda k m^{2} \exp (-2 k \sigma)}{3072 \pi}\left[\left(\Gamma_{1}+\Gamma_{2}\right) H+\frac{\dot{\Omega}_{1}}{\Omega_{1}} \Gamma_{1}+\frac{\dot{\Omega}_{2}}{\Omega_{2}} \Gamma_{2}\right]
\end{aligned}
$$

which represent the dissipation terms due to fermion-antifermion pairs production.

The complete system of differential equations describing the evolution in the inflationary phase we are interested in, includes also the equation ruling the evolution in time of the energy density of the relativistic fluid of $\Psi$ field quanta, which are produced via the decay term. This equation is provided by the covariant conservation of the energy-momentum tensor which reads

$$
d\left(a^{3} \rho_{R}\right)=-\left(p_{R}+p_{\sigma, \varphi_{c}}\right) d\left(a^{3}\right)-d\left(a^{3} \rho_{\sigma, \varphi_{c}}\right)
$$

where

$$
\begin{aligned}
& \rho_{\sigma, \varphi_{c}}=\frac{\dot{\varphi}_{c}^{2}}{2}+k \varphi_{c} \dot{\varphi}_{c} \dot{\sigma}+\frac{1}{2}\left[1+k^{2}\left(\varphi_{c}^{2}+\frac{Q^{2}}{4 \pi^{2}}\right)\right] \dot{\sigma}^{2}+V_{e f f}\left(\sigma, \varphi_{c}\right) \\
& p_{\sigma, \varphi_{c}}=\frac{\dot{\varphi}_{c}^{2}}{2}+k \varphi_{c} \dot{\varphi}_{c} \dot{\sigma}+\frac{1}{2}\left[1+k^{2}\left(\varphi_{c}^{2}+\frac{Q^{2}}{4 \pi^{2}}\right)\right] \dot{\sigma}^{2}-V_{e f f}\left(\sigma, \varphi_{c}\right)
\end{aligned}
$$

represent the pressure and the energy density of the total system of $\sigma-\varphi_{c}$ fields, respectively.

Equation (3.72), by using the definitions of $\rho_{\sigma, \varphi_{c}}$ and $p_{\sigma, \varphi_{c}}$ and the equations of motion (3.68), (3.69), can be cast in the form

$$
\dot{\rho}_{R}+4 H \rho_{R}=\gamma_{\sigma} \dot{\sigma}+\gamma_{\varphi_{c}} \dot{\varphi}_{c}
$$


Finally, for the Hubble parameter $H$ we get the expression

$$
H \equiv\left(\frac{\dot{a}}{a}\right)=\sqrt{\frac{2}{3}} k\left[\rho_{\sigma, \varphi_{c}}+\rho_{R}\right]^{1 / 2}
$$

The system of equations (3.68), (3.69), (3.75) and (3.76) allows to compute the $e$-fold number $N$ of the inflationary stage, defined as $N \equiv \log \left[a\left(t_{f}\right) / a(0)\right]$, where $t_{f}$ is the value of time at which the inflation ends. This time can be fixed as the one at which the radiation energy $\rho_{R}\left(t_{f}\right)$ eventually equals the energy density $\rho_{\sigma, \varphi_{c}}\left(t_{f}\right)$ stored in the background configuration $\varphi_{c}-\sigma$. In terms of the Hubble parameter (3.76), the expression for $N$ takes the simple form

$$
N=\int_{0}^{t_{f}} H\left(\sigma(t), \varphi(t), \rho_{R}(t)\right) d t
$$

The reheating temperature, namely the temperature at the beginning of radiation dominated era, can then be found by expressing the radiation energy density $\rho_{R}\left(t_{f}\right)$ in terms of the temperature. If one supposes in fact that a complete thermalization already occurred at $t_{f}$ due to fast gauge bosons mediated interactions among the fermions, we have

$$
\rho_{R}\left(t_{f}\right)=n_{f} \frac{\pi}{30} T_{R H}^{4}
$$

In Table 1 the $e$-fold $N$ and the reheating temperature are computed for some values of $Q$ and $\lambda$, with $f=1$, while in Figure 2 we report the trajectories in the $\sigma-\varphi_{c}$ plane for the same choices of the free parameters.

As already mentioned in the previous section the $\sigma-\varphi_{c}$ motion is initially characterized by a rolling down toward the minimum. During this phase, shown in Figure 2, the radiation energy density remains very small while the $e$-fold $N$ rapidly increases, reaching a value very close to the final one. From Table 1, one can see that its value depends quite strongly on the choice of $Q$ and $\lambda: N$ grows if $Q$ increases and/or $\lambda$ is small. In particular, with $Q=5 \div 10 M_{P l} N$ is of the order $10^{2}$, which is the value needed to solve the flatness problem of standard cosmology.

When the system reaches the absolute minimum of $V_{e f f}, \varphi_{c}$ starts oscillating around $\varphi_{c, m}$. For all cases reported in Table 1, for which the fermion coupling constant has been chosen to be quite large, $f=1$, the reheating temperature, $T_{R H}$, evaluated when 
$\rho_{\sigma, \varphi_{c}}=\rho_{R}$, is of the order of $10^{14} \div 10^{15} \mathrm{GeV}$. Interestingly this value is the one typical of GUT theory energy scales. If the value of $f$ is decreased, the reheating, in the one-loop approximation we have considered, becomes less efficient. In fact, already for $f \sim .1$ the energy injection from the $\sigma-\varphi_{c}$ system does not compensate the dilution of the radiation energy due to the universe expansion. Therefore, the value of the ratio $\rho_{R} / \rho_{\sigma, \varphi_{c}}$ reaches a maximum smaller than the unity, and then starts to decrease again. In these conditions the universe would continue to evolve in a $\rho_{\sigma, \varphi_{c}}$ matter dominated regime. This also happens for one of the cases reported in Table 1 , namely for the choice $Q=1$ and $\lambda=0.1$. The corresponding value for the $e$-fold number, which is however unsatisfactorily small, should be understood as the one at the beginning of the matter dominated era.

\section{Conclusions and remarks}

This paper represents a first step towards a systematic study of the cosmological implications of gauge theory and gravitation models inspired by noncommutative geometry. We have investigated this issue following [2, 4, 5], where gravitation and gauge theories are combined in the framework of noncommutative geometry. By virtue of the two-sheeted nature of the manifold, different scalar fields appear due to gravity and gauge connections. The scalar dynamics naturally provides a chaotic inflationary scenario in which, just after the Planck epoch, an initial field configuration $\left(\sigma(0), \varphi_{c}(0)\right)$ originates from quantum fluctuations with a corresponding energy density of the order of $M_{P l}^{4}$. The evolution from the above initial condition is then fixed by the theory. A remarkable aspect of this scenario is that the link between the scalar fields, the distance along the discrete dimension, and the role played by the gravity in the doubled manifold $\overline{\mathcal{M}}$, naturally fixes the initial values for $\sigma$ and $\varphi_{c}$. This contrasts with the arbitrariness of the usual models.

In this first analysis we have chosen an abelian gauge theory coupled with gravity, which represents the simplest model capturing all the essential ingredients of the dynamics. As is well-known, in noncommutative geometry to have a nontrivial scalar interaction (scalar potential) more than one fermion generation is needed.

We have considered the one-loop corrections to the classical potential due to the $\delta \varphi_{i}$ quantum fluctuations introducing a cut-off regularization; differently than in 10 
a renormalization procedure cannot be applied since the $\varphi_{c}$ vacuum expectation value explicitly depends on $\sigma$, as explained in section 3.1. Since from our numerical simulation it comes out that this field reaches its stationary point $\sigma_{m}$ quite soon, a similar procedure can be applied in fact for the subsequent evolution in which only the $\varphi_{c}$ field is still varying while $\sigma$ remains frozen at $\sigma_{m}$. To study the inflationary phase, however, since the main contribution to the $e$-fold number comes from the epoch in which $\sigma$ is rapidly evolving, we were forced to use the one-loop potential explicitly depending on the cut-off parameter $Q$, for which we have considered values of the order of the Planck mass.

The results show that, for reasonable values of the parameters, the value of $N$ is of the right order of magnitude $(N \sim 100)$ and the distance $d_{5}$ of the two sheets of the manifold $\overline{\mathcal{M}}$ remains of the order of the Planck length. It is interesting to note that it is the presence of the discrete dimension which is responsible for the inflationary expansion; this can be understood by realising that in the model it is $d_{5}^{-1}$ which play the role of the effective cosmological constant. It is possible, with different choices of the parameters, to have $d_{5}$ significantly growing as well, but this can only be done at the price of a much smaller $e$-fold.

The reheating phase has been studied by considering the contribution at one loop to the $\left\langle\delta \varphi_{i}^{2}\right\rangle$ of the interactions with fermion pairs. This introduces a dissipative term into the $\varphi_{c}$ and $\sigma$ equations of motion which describe, at this level of approximation, the energy release of the scalar fields to the relativistic degrees of freedom. We found that, provided the coupling constant is large enough, $f \sim 1$, a complete and efficient reheating is obtained and that the corresponding reheating temperature is of the order of $10^{14} \div 10^{15} \mathrm{GeV}$, well below the initial energy scale, which is set by the Planck scale. As we already pointed out, the approximation we have used is unsuitable for a detailed analysis of the reheating phenomenon. We can nevertheless conclude that there are strong indications of the presence of an efficient reheating stage.

There are at least two subjects which would be interesting to study further. First of all a more realistic model, based on a nonabelian gauge symmetry group, should be analyzed in more details, to see its implications for the inflationary scenarios. Incidentally the presence of couplings of the scalar fields with the gauge bosons should increase, in 
this case, the efficiency of the reheating mechanism.

It has been shown that in the model we have considered, the distance $d_{5}$ at the end of the inflationary era remains of the order of the Planck length; it remains an open question, however, if its subsequent evolution is non-trivial, namely that when the universe continues to expand after inflation, its value gradually increases, up to the inverse top quark mass, which is the value needed in order to reproduce the electroweak standard model. It seems that the idea of looking at $d_{5}$ as undergoing a dynamical evolution is quite important, since this could produce the onset of several phase transitions which took place during the expansion of the universe. 


\section{References}

[1] A. Connes, Noncommutative Geometry, Academic Press, 1994.

[2] A. Connes and J. Lott, Nucl. Phys. (Proc. Suppl.) B18 (1990) 29.

[3] J.C. Varilly and J.M. Gracia-Bondia, J. Geom. Phys. 12 (1993) 223.

[4] A.H. Chamseddine, G. Felder and J. Fröhlich, Phys. Lett. B296 (1993) 109; Nucl. Phys. B395 (1993) 672.

A.H. Chamseddine and J. Fröhlich, Phys. Rev. D50 (1994) 2893.

A.H. Chamseddine and J. Fröhlich, Phys. Lett. B314 (1993) 308.

B. Iochum and T. Schucker, Yang-Mills versus Connes-Lott, Marseille Preprint Cpt 94/P.3090, hep-th/9501142.

[5] A.H. Chamseddine, G. Felder and J. Fröhlich, Comm. Math. Phys. 155 (1993) 205.

A.H. Chamseddine and J. Fröhlich, Particle Physics Models, Grand Unification and Gravity in Non-Commutative Geometry, hep-th/9311068.

[6] G.Landi, N.A. Viet and C. Wali, Phys. Lett. B326 (1994) 45.

A. Sitarz, Class. Quant. Grav. 11 (1994) 2127.

N. Mohammedi, Mod. Phys. Lett. A9 (1994) 875.

[7] D. Kastler and T. Schucker, The Standard Model à la Connes-Lott, Marseille Preprint CPT-94/P.3901, hep-th/9412185, and references therein.

[8] E. Alvarez, J.M. Gracia-Bondia and C.P. Martin, Phys. Lett. B306 (1993) 55; Phys. Lett. B329 (1994) 259.

[9] S. Coleman and E. Weinberg, Phys. Rev. D7 (1973) 1888.

[10] A. Ringwald, Ann. of Phys. 177 (1987) 129.

[11] L. Kofman, A. Linde and A.A. Starobinsky, Phys. Rev. Lett. 73 (1994) 3195. 
[12] D. Boyanovsky, H.J. de Vega, R. Holman, D.-S. Lee and A. Singh, Phys. Rev. D51 (1995) 4419. 
Table 1.

\begin{tabular}{|c|c|c|c|}
\hline$Q$ & $\lambda$ & $N$ & $T_{R H}$ \\
$\left(M_{P l}\right)$ & & & $\left(10^{15} \mathrm{GeV}\right)$ \\
\hline 10 & 0.1 & 130 & 5. \\
5 & 0.01 & 110 & .6 \\
5 & 0.1 & 40 & 2. \\
1 & 0.01 & 20 & .1 \\
1 & 0.1 & 10 & no reheat. \\
\hline
\end{tabular}




\section{Figure Captions}

Figure 1. The effective potential $V_{\text {eff }}\left(\sigma, \varphi_{c}\right)$ is plotted for $\lambda=.1$ and $Q=10 M_{P l}$ and $m=$ $1 M_{P l}$.

Figure 2. The trajectories in the $\sigma-\varphi_{c}$ plane corresponding to the values of the parameters reported in Table 1, are shown. The labels refer to the order in which they are considered in the Table, from top to bottom.

\section{Table Caption}

Table 1. The $e$-fold number, $N$, and the reheating temperature, $T_{R H}$, are shown for some choices of the parameters $Q, \lambda$ (the values correspond to $f=1$.). 


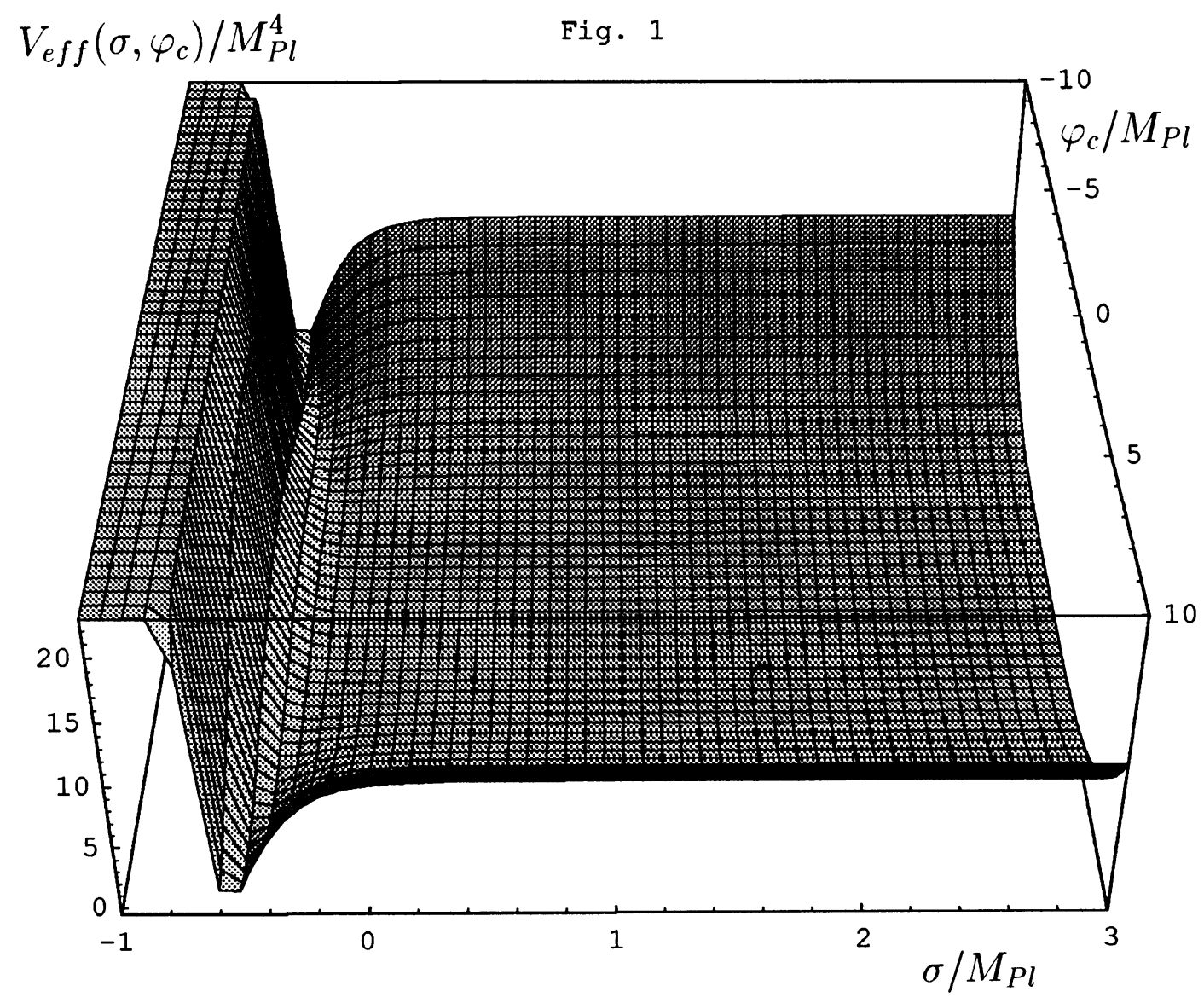




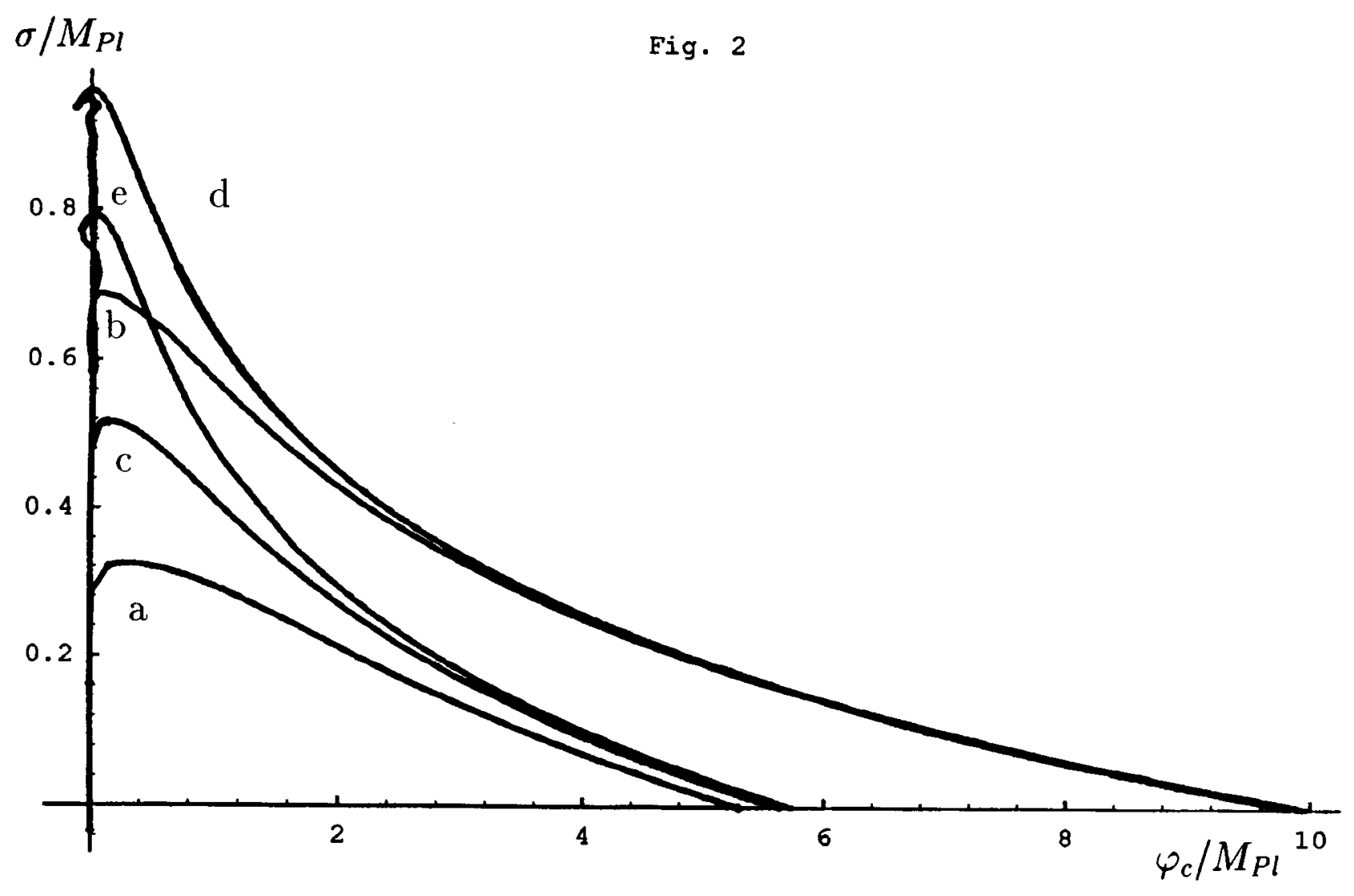

